\title{
Local fluctuations in the ageing of a simple structural glass
}

\author{
HORACIO E. CASTILLO* AND AZITA PARSAEIAN \\ Department of Physics and Astronomy, Ohio University, Athens, Ohio 45701, USA \\ *e-mail: castillo@phy.ohiou.edu
}

The presence of dynamical heterogeneities-that is, nanometre-scale regions of molecules rearranging cooperatively at very different rates compared with the bulk ${ }^{1,2}$-is increasingly being recognized as crucial to our understanding of the glass transition, from the non-exponential relaxation to the divergence of the relaxation times ${ }^{3}$. Although recent experiments ${ }^{4-11}$ and simulations ${ }^{12-14}$ have observed their presence directly, a clear physical picture of their origin is still lacking. Here, we present the first detailed characterization of the statistics of local fluctuations in a simulation of the ageing of a continuousspace, quasi-realistic structural glass model. A possible physical mechanism $^{15-18}$ for the origin of dynamical heterogeneities in the non-equilibrium dynamics of glassy systems predicts universal scaling of the probability distributions of twotime local fluctuations. We find that to a first approximation this scaling is indeed satisfied by our results. We propose to test our results using confocal microscopy and atomic force microscopy experiments.

Supercooled liquids approaching the glass transition show increasingly slow dynamics, until eventually they cannot equilibrate in laboratory timescales ${ }^{19}$. One consequence of this fact is physical ageing, that is, the breakdown of time translation invariance: the correlation $C\left(t, t_{\mathrm{w}}\right)$ between spontaneous fluctuations of an observable at times $t$ (the final time) and $t_{\mathrm{w}}$ (the waiting time) is a non-trivial function of $t$ and $t_{\mathrm{w}}$, as opposed to being a function of the time difference $t-t_{\mathrm{w}}$. In many cases, the two-time correlation $C\left(t, t_{\mathrm{w}}\right)$ in an ageing system separates into a fast, time-translationinvariant contribution $C_{\text {fast }}\left(t-t_{\mathrm{w}}\right)$ and a slow contribution $C_{\text {slow }}\left(t, t_{\mathrm{w}}\right)$ (ref. 20): $C\left(t, t_{\mathrm{w}}\right)=C_{\text {fast }}\left(t-t_{\mathrm{w}}\right)+C_{\text {slow }}\left(t, t_{\mathrm{w}}\right)$. For some systems, the slow part of the correlation has the form ${ }^{20}$ $C_{\text {slow }}\left(t, t_{\mathrm{w}}\right)=C_{\text {slow }}\left(h(t) / h\left(t_{\mathrm{w}}\right)\right)$, where $h(t)$ is some monotonically increasing function. For example, in the case of domain growth, $h(t)$ is proportional to the domain size ${ }^{20}$. In what follows, only the slow part of the correlation is considered, and any effects due to the fast part of the dynamics are ignored.

Recently, it has been proven that, in the limit of long times, the dynamics of a class of spin-glass models is invariant under global reparametrizations $t \rightarrow h(t)$ of the time ${ }^{15}$. This result has been used to predict the existence of a Goldstone mode in the non-equilibrium dynamics, associated with smoothly varying local fluctuations in the reparametrization of the time $t \rightarrow h_{\mathrm{r}}(t)=\mathrm{e}^{\varphi_{\mathrm{r}}(t)}$ (refs 16,17). These fluctuations have been physically interpreted to represent local fluctuations of the age of the sample ${ }^{16,17}$. In the cases where the global two-time correlation shows $h(t) / h\left(t_{\mathrm{w}}\right)$ scaling, a simple Landau-theory approximation for the dynamical action predicts $^{16-18}$ that the full probability distribution $\rho\left(C_{\mathrm{r}}\left(t, t_{\mathrm{w}}\right)\right)$ of local correlations $C_{\mathrm{r}}\left(t, t_{\mathrm{w}}\right)$ depends on the times $t, t_{\mathrm{w}}$ only through the values of the global correlation $C_{\text {global }}\left(t, t_{\mathrm{w}}\right)$. However, this scaling of $\rho\left(C_{\mathrm{r}}\left(t, t_{\mathrm{w}}\right)\right)$ with $C_{\text {global }}\left(t, t_{\mathrm{w}}\right)$ was also found in the coarsening dynamics of the $O(N)$ ferromagnet, where the time reparametrization symmetry is not present ${ }^{21}$. It is not known whether off-lattice, quasi-realistic models, describing structural glasses, show the same time reparametrization symmetry as spin glasses, or whether their dynamics shows any evidence of the Goldstone mode associated with this symmetry. A first test for this statement would be to check whether $\rho\left(C_{\mathrm{r}}\left(t, t_{\mathrm{w}}\right)\right)$ scales with $C_{\text {global }}\left(t, t_{\mathrm{w}}\right)$. If this test fails, then the presence of the time reparametrization symmetry can be excluded.

Simulations of fluctuations in glass-forming liquids have mostly focused on the (equilibrium) supercooled liquid, and on determining the spatial correlation of fluctuations between different points in space ${ }^{12-14}$. In ref. 22 the ageing regime was studied, but only the spatial correlations of fluctuations were measured, and in ref. 18 spin-glass and kinetically constrained lattice models were studied.

Here, we present the first detailed characterization of the statistics of local fluctuations in the ageing of a continuous-space, quasi-realistic structural glass model. Numerically simulating the (non-equilibrium) ageing regime allows us to address many experiments working in this regime that probe dynamical heterogeneities microscopically ${ }^{7-11}$. We focus here on determining the statistical distribution of fluctuations at one point in space, for various reasons: (1) to make direct contact with experiments using local probes to study dynamical heterogeneities, which also obtain this kind of distribution ${ }^{5,6,10}$; (2) to obtain additional physical information beyond the second moment of the fluctuations and (3) to test whether the probability distribution of local fluctuations in the ageing regime depends on times only through the value of $C_{\text {global }}\left(t, t_{\mathrm{w}}\right)$.

We probe individual particle displacements along one direction $\Delta x_{j}\left(t, t_{\mathrm{w}}\right)=x_{j}(t)-x_{j}\left(t_{\mathrm{w}}\right)$ (where $j$ is the particle index), and also local, coarse-grained two-time functions: the correlator

$$
C_{\mathbf{r}}\left(t, t_{\mathrm{w}}\right)=\frac{1}{N\left(B_{\mathbf{r}}\right)} \sum_{\mathbf{r}_{j}\left(t_{\mathrm{w}}\right) \in B_{\mathbf{r}}} \cos \left(\mathbf{q} \cdot\left(\mathbf{r}_{j}(t)-\mathbf{r}_{j}\left(t_{\mathrm{w}}\right)\right)\right),
$$

and the mean square displacement

$$
\Delta_{\mathbf{r}}\left(t, t_{\mathrm{w}}\right)=\frac{1}{N\left(B_{\mathbf{r}}\right)} \sum_{\mathbf{r}_{j}\left(t_{\mathrm{w}}\right) \in B_{\mathbf{r}}}\left(\mathbf{r}_{j}(t)-\mathbf{r}_{j}\left(t_{\mathrm{w}}\right)\right)^{2} .
$$

Here we consider a coarse-graining cubic-shaped box $B_{\mathrm{r}}$ of side $l$ around the point $\mathbf{r}$ in the system, and the sums run over the $N\left(B_{\mathbf{r}}\right)$ particles present at the waiting time $t_{\mathrm{w}}$ in $B_{\mathrm{r}}$. We choose a value of 

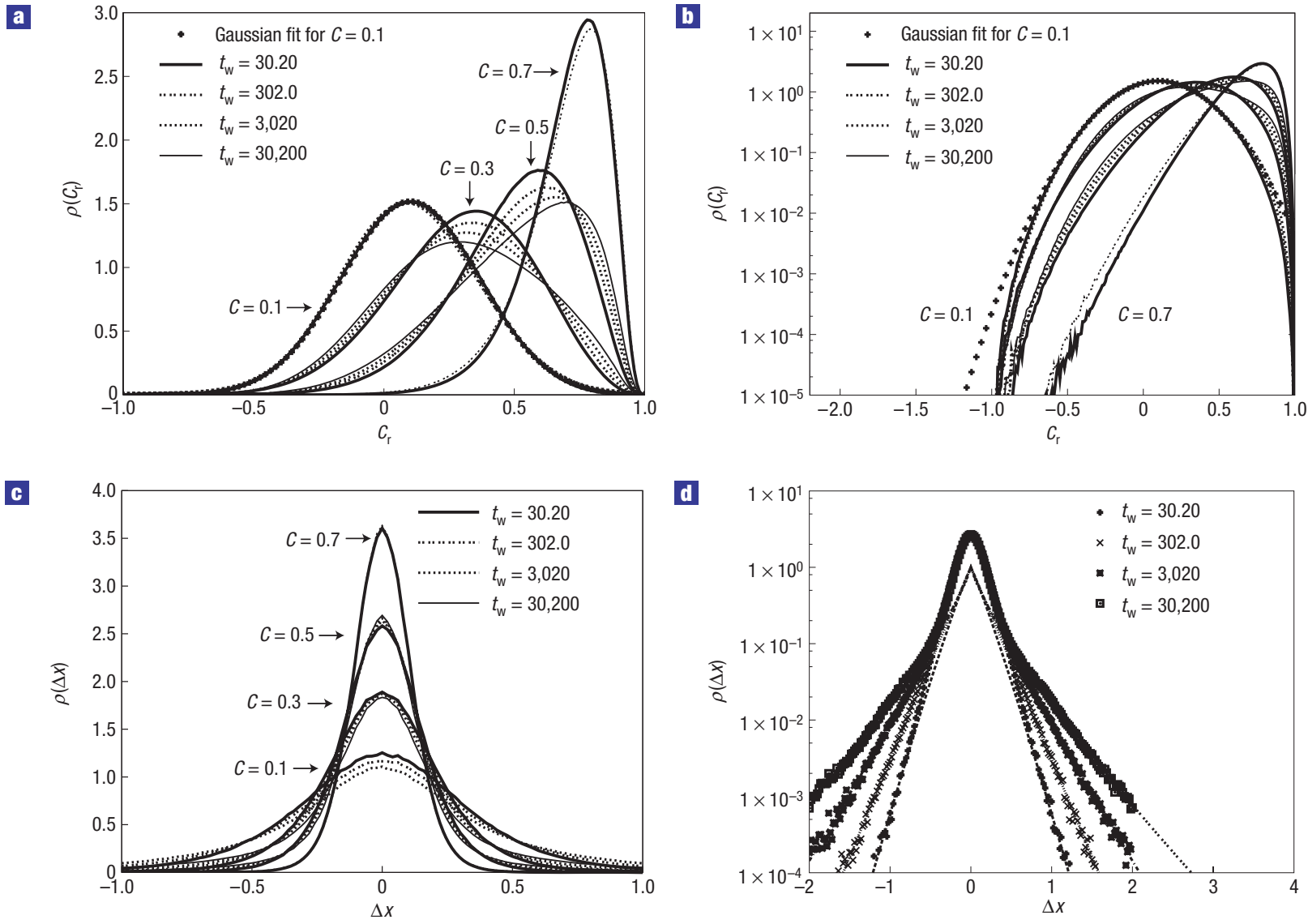

Figure 1 Probability distributions. a,b, $\rho\left(C_{\mathrm{r}}\left(t, t_{\mathrm{w}}\right)\right)$ measured for $30.20 \leq t_{\mathrm{w}} \leq 30,200$, plotted for final times $t$ chosen so that $C_{\text {global }}\left(t, t_{\mathrm{w}}\right) \in\{0.1,0.3,0.5,0.7\}$. Coarse graining size $I \approx 0.11 L$ (with $L \equiv$ linear size of the simulation box). The curves collapse into four groups, corresponding to $C_{\text {global }}=0.7,0.5,0.3,0.1$ (ordered from highest to lowest value of $C_{\mathrm{r}}$ at the peak). A gaussian fit to the data for $C=0.1$ is also shown. Linear scale (a). Logarithmic scale (b). c, $\rho\left(\Delta x\left(t, t_{\mathrm{w}}\right)\right)$ for $30.20 \leq t_{\mathrm{w}} \leq 30,200$, with $t$ chosen so that $C_{\text {global }}\left(t, t_{\mathrm{w}}\right) \in\{0.1,0.3,0.5,0.7\}$. The curves collapse into four groups, corresponding to $C_{\text {global }}=0.1,0.3,0.5,0.7 . \mathbf{d}$, Tails of $\rho\left(\Delta x\left(t, t_{\mathrm{w}}\right)\right)$, for $C=0.5$ and $t_{\mathrm{w}}=30.20,302,3,020,30,200$ (from narrower to wider tail). Symbols: results from simulation. Lines: fits to the data for $|\Delta x|>0.5$ using

$\rho\left(\Delta x\left(t, t_{\mathrm{w}}\right)\right) \approx N \exp \left(-|\Delta x / a|^{\beta}\right)$.

$q$ that corresponds to the main peak in the structure factor $S(q)$ of the system, $q=7.2$ in Lennard-Jones (LJ) units.

These definitions are inspired by the analogous definitions in the case of spin glasses ${ }^{16,17}$, and can be applied to analyse data obtained both from simulations and from confocal microscopy experiments. The global quantities $C_{\text {global }}\left(t, t_{\mathrm{w}}\right)$ (incoherent part of the intermediate scattering function) and $\Delta_{\text {global }}\left(t, t_{\mathrm{w}}\right)$ (mean square displacement) are defined by extending the sum to the whole system in equations (1) and (2) respectively.

We carried out 250 independent molecular-dynamics runs for the binary LJ system of ref. 23, which has a mode-coupling critical temperature $T_{c}=0.435$. A system of 8,000 particles was equilibrated at a temperature $T_{0}=5.0$, then instantly quenched to $T=0.4$, and finally it was allowed to evolve for $10^{5} \mathrm{LJ}$ time units. The origin of times was taken at the instant of the quench.

In Fig. 1a,b we present our results for the probability distribution $\rho\left(C_{\mathrm{r}}\left(t, t_{\mathrm{w}}\right)\right)$ of the local intermediate scattering function for waiting times $t_{\mathrm{w}}=30.20, \ldots, 30,200$, and final times $t$ chosen so that $C_{\text {global }}\left(t, t_{\mathrm{w}}\right) \in\{0.1,0.3,0.5,0.7\}$. We observe that the data approximately collapse for each value of $C_{\text {global }}\left(t, t_{\mathrm{w}}\right)$ (a less clear collapse is observed at constant $\Delta_{\text {global }}\left(t, t_{\mathrm{w}}\right)$; details of this comparison will be presented elsewhere). This collapse at constant $C_{\text {global }}\left(t, t_{\mathrm{w}}\right)$ is also observed in simulations in a three-dimensional spin-glass model, but in the case of the spin-glass model the collapse is more precise than here. Unlike the case of the three-dimensional spin-glass model, the position of the peak in the distribution $\rho\left(C_{\mathrm{r}}\right)$ is strongly dependent on the value of $C_{\text {global }}\left(t, t_{\mathrm{w}}\right)$. The distribution $\rho\left(C_{\mathrm{r}}\left(t, t_{\mathrm{w}}\right)\right)$ evolves gradually from being highly skewed and non-gaussian for $C_{\text {global }}\left(t, t_{\mathrm{w}}\right)=0.7$ to being unskewed and very close to gaussian for $C_{\text {global }}\left(t, t_{\mathrm{w}}\right)=0.1$. Notice here that the distributions of local observables are also expected to become more gaussian as $C_{\text {global }}\left(t, t_{\mathrm{w}}\right)$ is increased beyond $C_{\text {global }}\left(t, t_{\mathrm{w}}\right) \approx 0.7$, that is, in the quasi-equilibrium regime corresponding to the first step in the two-step relaxation. This is indeed observed in experiments probing fluctuations in dipole moments of nanometre-scale regions ${ }^{11}$ and also in our simulations, in the probability distributions $\rho(\Delta x)$ of onedimensional displacements.

To characterize the weak dependence of the probability distributions on waiting time at fixed $C_{\text {global }}\left(t, t_{\mathrm{w}}\right)$, in Fig. 2a we plot the centred second moment of the distributions $\rho\left(C_{\mathrm{r}}\right)$ as a function of waiting time, for fixed $C_{\text {global }}\left(t, t_{\mathrm{w}}\right) \in\{0.1,0.3,0.5,0.7\}$. The dependence on $t_{\mathrm{w}}$ is so weak that both a logarithmic form and a power-law form (with powers in the range 0.01-0.07) provide a good fit. We can explain the fact that $\rho\left(C_{\mathrm{r}}\right)$ does show some dependence on $t_{\mathrm{w}}$ for fixed $C_{\text {global }}$ by the presence of a 


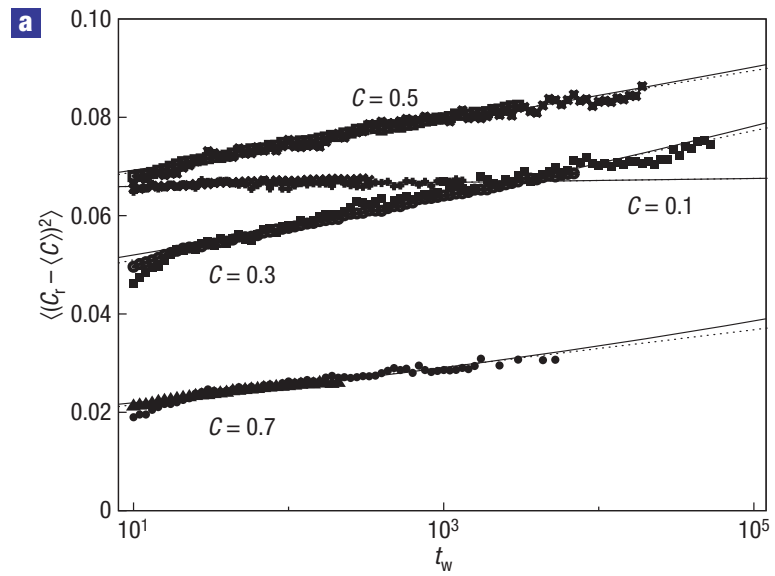

b

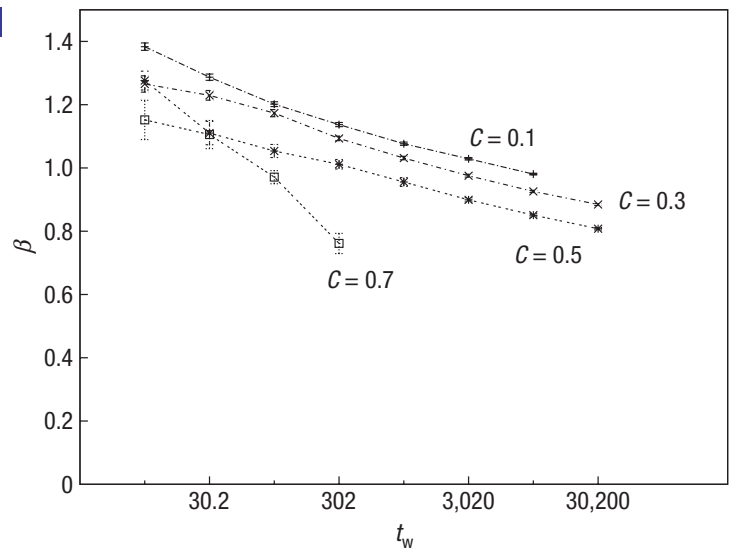

Figure 2 Dependence of probability distributions on the waiting time. Evolution of the probability distributions, as a function of $t_{\mathrm{w}}$, at constant $C_{\text {global }}\left(t, t_{\mathrm{w}}\right) \in\{0.1,0.3,0.5,0.7\}$. a, Second moment of $\rho\left(C_{\mathrm{r}}\left(t, t_{\mathrm{w}}\right)\right)$, together with fits to the functional forms: $m_{0}\left(t_{\mathrm{w}}\right)^{a}$ (full lines) and $m_{0}^{\prime} \log \left(t_{\mathrm{w}} / t_{0}\right)$ (dotted lines). b. Stretching exponent $\beta$ for the tails of $\rho(\Delta x)$, as a function of the waiting time $t_{\mathrm{w}}$, at constant $C_{\text {global }}\left(t, t_{\mathrm{w}}\right) \in\{0.1,0.3,0.5,0.7\}$ (the lines are guides to the eye). The error bars indicate the statistical errors in $\beta$.

time-dependent dynamic correlation length. As in the case of simulations of spin glasses ${ }^{17}$, the dynamic correlation length in the present system grows very slowly as a function of $t_{\mathrm{w}}$, but for the timescales of the simulation it is not yet larger than the size of the coarse-graining box used ${ }^{24}$. Thus, some of the fluctuations are averaged out, and the width of the distribution is reduced. This effect is stronger for shorter $t_{\mathrm{w}}$, consistent with the trend shown in Fig. 2a.

In Fig. 1c,d, we present our results for the probability distribution $\rho\left(\Delta x\left(t, t_{\mathrm{w}}\right)\right)$ of the particle displacements $\Delta x_{j}\left(t, t_{\mathrm{w}}\right)=x_{j}(t)-x_{j}\left(t_{\mathrm{w}}\right)$ along one direction. In Fig. 1c, we can observe that these data also approximately collapse for each value of $C_{\text {global }}\left(t, t_{\mathrm{w}}\right)$. In Fig. $1 \mathrm{~d}$, we have a closer look at the tails of $\rho\left(\Delta x\left(t, t_{\mathrm{w}}\right)\right)$. We find that the distribution is nongaussian, as was observed in experiments in colloidal glasses in the supercooled regime $e^{5,6}$. We can fit the tails of the distribution with a nonlinear exponential form $\rho(\Delta x) \approx N \exp \left(-|\Delta x / a|^{\beta}\right)$, and they become more prominent as $t_{\mathrm{w}}$ grows (for constant $C_{\text {global }}\left(t, t_{\mathrm{w}}\right)$ ). Indeed, as shown in Fig. 2b, the exponent $\beta$ decreases from $\beta>1$ ('compressed exponential') at short $t_{\mathrm{w}}$ to $\beta \approx 0.8$ ('stretched exponential') at much longer $t_{\mathrm{w}}$.

To summarize, we have presented the first detailed characterization of the probability distributions of non-equilibrium fluctuations in the ageing regime in a continuous-space, quasi-realistic structural glass model. Our main result is that the probability distributions for the local fluctuating two-time quantities are, to a first approximation, invariant when the global intermediate scattering function $C_{\text {global }}\left(t, t_{\mathrm{w}}\right)$ is kept constant. This behaviour is similar to the behaviour found in the non-equilibrium dynamics of short-range spin-glass models ${ }^{16,17}$ and some kinetically constrained lattice models ${ }^{18}$, and in the coarsening dynamics of the $O(N)$ model $^{21}$. As a consequence, our results cannot rule out the presence of a Goldstone mode associated with local fluctuations in the age of the sample, but alternative interpretations are still possible ${ }^{21}$. Besides this simple scaling, our results provide detailed predictions for the statistical properties of fluctuations in ageing structural glasses. These predictions can be directly tested by applying a similar analysis to experimental data from confocal microscopy in colloidal glass systems ${ }^{5-7}$, and also possibly by analysing atomic force microscopy experiments probing nanoscale polarization fluctuations $s^{8-11}$.

Received 11 May 2006; accepted 30 October 2006; published 24 December 2006.

\section{References}

1. Ediger, M. D. Spatially heterogeneous dynamics in supercooled liquids. Annu. Rev. Phys. Chem. 51, 99-128 (2000).

2. Sillescu, H. Heterogeneity at the glass transition: A review. J. Non-Cryst. Solids 243, 81-108 (1999).

3. Adam, G. \& Gibbs, J. H. On the temperature dependence of cooperative relaxation properties in glass-forming liquids. J. Chem. Phys. 43, 139-146 (1965).

4. Kegel, W. K. \& Blaaderen, A. V. Direct observation of dynamical heterogeneities in colloidal hard-sphere suspensions. Science 287, 290-293 (2000).

5. Weeks, E. R., Crocker, J. C., Levitt, A. C., Schofield, A. \& Weitz, D. A. Three-dimensional direct imaging of structural relaxation near the colloidal glass transition. Science 287, 627-631 (2000).

6. Weeks, E. R. \& Weitz, D. A. Properties of cage rearrangements observed near the colloidal glass transition. Phys. Rev. Lett. 89, 095704 (2002).

7. Courtland, R. E. \& Weeks, E. R. Direct visualization of aging in colloidal glasses. J. Phys. C 15, S359-S365 (2003).

8. Vidal-Russell, E., Israeloff, N. E., Walther, L. E. \& Alvarez Gomariz, H. Nanometer scale dielectric fluctuations at the glass transition. Phys. Rev. Lett. 81, 1461-1464 (1998).

9. Walther, L. E., Israeloff, N. E., Vidal-Russell, E. \& Alvarez Gomariz, H. Mesoscopic-scale dielectric relaxation at the glass transition. Phys. Rev. B 57, R15112-R15115 (1998).

10. Vidal-Russell, E. \& Israeloff, N. E. Direct observation of dynamical heterogeneities in colloidal hard-sphere suspensions. Nature 408, 695-698 (2000).

11. Sinnathamby, K. S., Oukris, H. \& Israeloff, N. E. Local polarization fluctuations in an aging glass. Phys. Rev. Lett. 95, 067205 (2005).

12. Glotzer, S. C. Spatially heterogeneous dynamics in liquids: insights from simulation. J. Non-Cryst. Solids 274, 342-355 (2000).

13. Kob, W., Donati, C., Plimpton, S. J., Poole, P. H. \& Glotzer, S. C. Dynamic heterogeneities in supercooled Lennard-Jones liquid. Phys. Rev. Lett. 79, 2827-2830 (1997).

14. Lacevic, N., Starr, F. W., Schroder, T. B. \& Glotzer, S. C. Spatially heterogeneous dynamics investigated via a time-dependent four-point density correlation function. J. Chem. Phys. 119, 7372-7387 (2003).

15. Chamon, C., Kennett, M. P., Castillo, H. E. \& Cugliandolo, L. F. Separation of time scales and reparametrization invariance for aging systems. Phys. Rev. Lett. 89, 217201 (2002).

16. Castillo, H. E., Chamon, C., Cugliandolo, L. F. \& Kennett, M. P. Heterogeneous aging in spin glasses. Phys. Rev. Lett. 88, 237201 (2002).

17. Castillo, H. E., Chamon, C., Cugliandolo, L. F., Iguain, J. L. \& Kennett, M. P. Spatially heterogeneous ages in glassy systems. Phys. Rev. B 68, 134442 (2003).

18. Chamon, C., Charbonneau, P., Cugliandolo, L. F., Reichman, D. R. \& Sellitto, M. Out-of-equilibrium dynamical fluctuations in glassy systems. J. Chem. Phys. 121, 10120-10137 (2004).

19. Zallen, R. The Physics of Amorphous Solids (Wiley, New York, 1983).

20. Bouchaud, J.-P., Cugliandolo, L. F., Kurchan, J. \& Mézard, M. in Spin Glasses and Random Fields (ed. Young, A. P.) 161-224 (World Scientific, Singapore, 1998).

21. Chamon, C., Cugliandolo, L. F. \& Yoshino, H. Fluctuations in the coarsening dynamics of the $\mathrm{O}(\mathrm{N})$ model with $\mathrm{N} \rightarrow \infty$ : are they similar to those in glassy systems? J. Stat. Mech. P01006 (2006).

22. Parisi, G. An increasing correlation length in off-equilibrium glasses. J. Phys. Chem. B 103, 4128-4131 (1999).

23. Kob, W. \& Barrat, J.-L. Aging effects in a Lennard-Jones glass. Phys. Rev. Lett. 78, 4581-4584 (1997)

24. Parsaeian, A. \& Castillo, H. E. Growth of spatial correlations in the aging of a simple structural glass. Preprint at $<$ http://arxiv.org/abs/cond-mat/0610789> (2006).

\section{Acknowledgements}

H.E.C. especially thanks C. Chamon and L. Cugliandolo for very enlightening discussions over the years and J. P. Bouchaud, S. Glotzer, N. Israeloff, M. Kennett, D. Reichman and E. Weeks for suggestions and discussion. This work was supported in part by the DOE under grant DE-FG02-06ER46300, by the NSF under grant PHY99-07949 and by Ohio University. Numerical simulations were carried out at the Ohio Supercomputing Center and at the Boston University SCV.

H.E.C. acknowledges the hospitality of the Aspen Center for Physics.

Correspondence and requests for materials should be addressed to H.E.C.

Competing financial interests

The authors declare that they have no competing financial interests.

Reprints and permission information is available online at http://npg.nature.com/reprintsandpermissions/ 